\title{
Cefazolin potency against methicillin-resistant Staphylococcus aureus: a microbiologic assessment in support of a novel drug delivery system for skin and skin structure infections [Erratum]
}

Nicolau DP, Silberg BN. Infect Drug Resist. 2017;10:227-230.

Page 227, affiliations list, the text "Department of Surgery, Sonoma West Medical Center, Sebastopol, CT, USA" should read "Department of Surgery, Sonoma West Medical Center, Sebastopol, CA, USA". 